\title{
Clinical Approaches to Breast Reconstruction: What Is the Appropriate Reconstructive Procedure for My Patient?
}

\author{
Max Dieterich $^{a}$ Adrian Dragu $^{\mathrm{b}} \quad$ Angrit Stachs $^{\mathrm{a}} \quad$ Johannes Stubert $^{\mathrm{a}}$

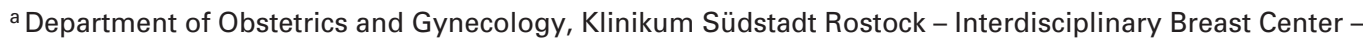 \\ University of Rostock, Rostock, Germany; \\ ${ }^{b}$ Department of Plastic and Hand Surgery, Carl Gustav Carus University of Dresden, Dresden, Germany
}

\section{Keywords}

Breast reconstruction - Heterologous breast reconstruction - Autologous breast reconstruction . Mesh · Acellular dermal matrix

\section{Summary}

Breast reconstruction after breast cancer is an emotional subject for women. Consequently, the correct timing and surgical procedure for each individual woman are important. In general, heterologous or autologous reconstructive procedures are available, both having advantages and disadvantages. Breast size, patient habitus, and previous surgeries or radiation therapy need to be considered, independent of the chosen procedure. New surgical techniques, refinement of surgical procedures, and the development of supportive materials have increased the general patient collective eligible for breast reconstruction. This review highlights the different approaches to immediate breast reconstruction using autologous or heterologous techniques.

(C) 2017 S. Karger GmbH, Freiburg

\section{Introduction}

Breast cancer is the most common cancer in women with approximately 182,000 new cases annually in the USA and 70,000 new cases in Germany [1]. Around 17,500 women die of breast cancer each year in Germany, whereas the 10-year survival rate is about $82 \%$ for all breast cancer entities. Over the last decade, major developments have led to improved survival rates. With the introduction of neoadjuvant chemotherapy, which today is a widely ac- cepted concept, mastectomy rates have further decreased. Nevertheless, in about $20-30 \%$ of all breast cancer cases, a mastectomy is indicated (table 1). To protect women from the psychosocial stress associated with the loss of a breast and compromised female integrity (see accompanying article in this edition), immediate breast reconstruction can be offered. However, breast reconstruction is not always easy, and not infrequently various patient-related aspects have to be considered to decide for or against a reconstructive concept. This contribution will focus on the clinical approaches to immediate breast reconstruction, whereas secondary breast reconstruction will only be touched upon.

Since the proven safety of skin- and nipple-sparing mastectomy (SSM, NSM), reconstructive options for women with breast cancer have increased [2-4]. For breast reconstruction, the correct timing and patient selection are crucial to achieve satisfying aesthetic results. Always keeping in mind that oncology safety comes first, prior to reconstruction, the indication for postmastectomy radiation therapy (PMRT) needs to be ruled out, as radiation therapy decreases the aesthetic result of implant-based as well as autologous breast reconstruction $[4,5]$. Today, PMRT is a relative indication in cases of 1-3 positive lymph nodes and obligatory in $\geq 4$ lymph nodes [6-8]. Consequently, the sentinel lymph node should be examined in a primary surgery. Alternatively, the lymph node may be examined in the same surgical setting via intraoperative frozen section while accepting the risk of potentially having to irradiate the reconstructed breast in the case of a positive SLN and keeping in mind that sensitivity rates of intraoperative sentinel lymph node evaluation range from 58 to $72 \%$ [9-11]. Where PMRT is indicated, reconstruction should be delayed, and secondary autologous breast reconstruction is the preferred reconstructive approach. Nevertheless, some patients insist on immediate breast reconstruction, even in cases of a known indication for PMRT. Reasons for this are diverse and can be subsumed under the term of 'postmastectomy syndrome'. These cases must be discussed individually, and adequate

\section{KARGER}

() 2017 S. Karger GmbH, Freiburg

Fax +497614520714 
information about risks and complications is mandatory. To maintain the skin envelop in these cases, a temporary expander or implant can be placed under the skin or under the chest wall muscle during the time of irradiation. The advantage of this strategy is that skin can be spared, thus helping to improve the outcome at the time of secondary reconstruction (heterologous or autologous). Controversy exist as to whether to irradiate the expander or the implant [12-15]. Regarding the aesthetic outcome, no general recommendations can be made. Capsular contraction, tissue fibroses, and atrophy are mediated by PMRT in both implant and autologous breast reconstruction [16-19].

\section{Before Breast Reconstruction}

Today, more women with an indication for mastectomy - even after neoadjuvant chemotherapy - are interested in breast reconstruction. With new techniques and improved surgical skills, breast reconstruction can be offered to a wider range of women. Consequently, also patients with challenging anatomical shapes like very small, very large, or ptotic breasts, or a combination thereof may demand immediate breast reconstruction. Prior to deciding on a reconstructive approach, several points need to be considered. During the consultation, the patient's habitus, breast volume, shape, body weight, and wishes need to be taken into account. Preand postoperative results of 'similar' cases should be presented to give an impression of realistic results. A rather 'ideal' patient for immediate reconstruction is a woman who had enough time to dis-

Table 1. Indications for mastectomy

Table 2. Clinical consideration and selection criteria

Inflammatory disease
Large DCIS (ductal carcinoma in situ) alone
Invasive carcinoma with large accompanying DCIS
Prophylactic mastectomy
Wish for mastectomy
Poor breast/tumor size ratio

Consider patient habitus

Careful medical history

Evaluation of comorbidities

Give information regarding reconstructive options (autologous vs. implant; pedicled vs. free flap)

Demonstration of realistic postoperative results (photo documentation, aspects of symmetry)

Discussion of patient expectations

Point out possible complications and consequences cuss the different approaches and techniques, is a non-smoker, non-obese, and psychologically stable. Psychological factors comprise especially the evaluation of the patient's expectations (realistic and unrealistic), which are, besides the surgical skills, essential for satisfying results. Diabetes is a relative contraindication, but in combination with other comorbidities it may be considered a definite contraindication (table 2).

\section{Autologous versus Heterologous Reconstruction}

Over the last decade, the philosophy regarding autologous versus heterologous reconstruction has undergone a transition [20]. In the past, patients with smaller breast were rather candidates for implant-based and patients with bigger breasts for autologous reconstruction. Today, almost every breast can be reconstructed with both procedures, taking the goals and expectations of the patient into account. Advancements in microsurgery, development of new donor sites, as well as new techniques in implant reconstruction and the introduction of additional materials like acellular dermal matrices $(\mathrm{ADM})$ and meshes are fundamental developments responsible for this change [21]. Nevertheless, a classification for certain patient characteristics and reconstructive approaches will be performed for didactic reasons.

\section{Primary Breast Reconstruction}

\section{Implant-Based Breast Reconstruction (Slim Patients)}

The simplest and most common method of breast reconstruction is implant-based breast reconstruction. In around $70-80 \%$ of all immediate breast reconstructions, implants are used [22]. Slim patients with small and rather round breasts are good candidates for immediate implant-based breast reconstruction. During surgery, the inframammary fold (IMF) needs to be marked bilaterally, helping to place the implant in the correct position. Usually an anatomical form-stable silicone implant is used in this collective. In very small, flat, and non-ptotic breasts, round implants may be considered. A contralateral procedure is not required in most cases or can be considered later if necessary. The surgical approach is usually performed using a tennis racquet, croquet-like, periareolar, or inframammary incision. Usually, a direct-to-implant procedure is possible (fig. 1).

In some cases, additional materials like ADM or synthetic meshes are needed to support the reconstructive procedure [23,

Fig. 1. 41-year-old patient with multifocal invasive lobular carcinoma, left breast. Skin-sparing mastectomy with subpectoral direct-to-implant breast reconstruction (Allergan Style 410LL, $135 \mathrm{~g}$ ). 2 weeks postoperatively.

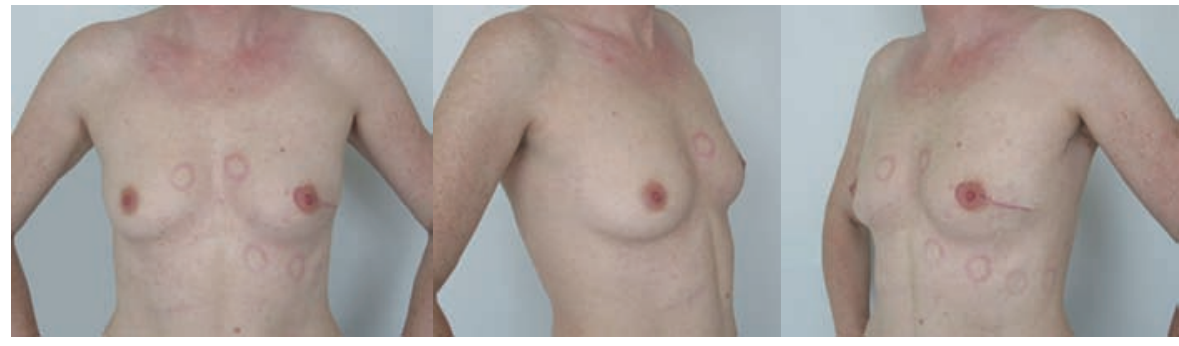


24]. Although ADM are used in almost 50\% of all implant procedures in the USA, in our practice, these supportive materials are less frequently necessary $[25,26]$.

In slim patients with bigger breasts, the clinical approach differs and a contralateral procedure is usually needed. In many cases, the affected breast needs a breast lift in addition to the implant. This is due to the limited space in the retropectoral pocket compared to the resected prepectoral breast mount, resulting in excessive skin. Usually, an inverted $\mathrm{T}$ incision is used analog to a Wise-pattern breast reduction. This has the decisive advantage of preparing a dermal flap supporting the inferior part of the implant

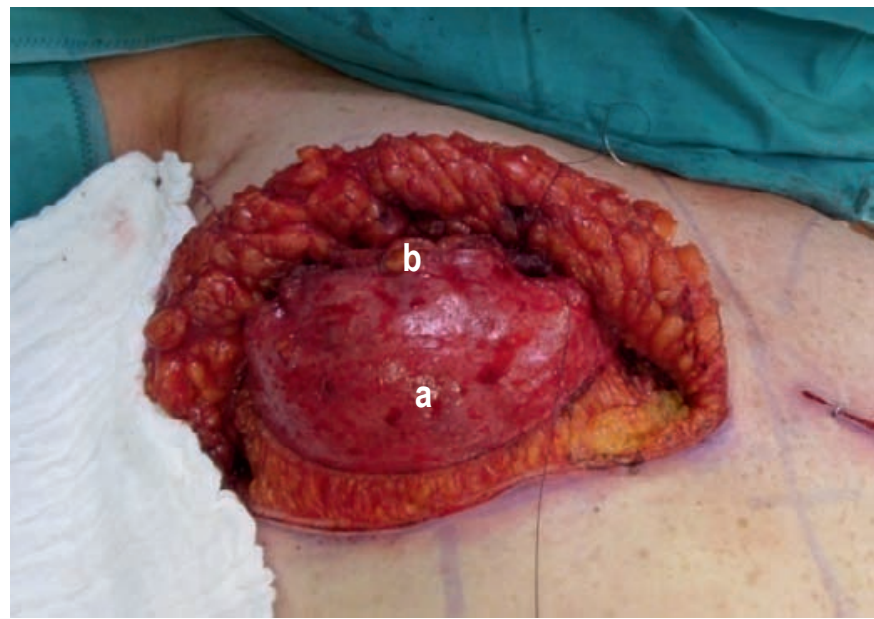

Fig. 2. Intraoperative view of a skin-sparing mastectomy, right side, using an inverted $\mathrm{T}$ incision. An inferior dermal flap (a) was resected for additional support and sutured to the inferior pole of the pectoralis major muscle (b). No mesh or acellular dermal matrices needed. pocket and defining the new IMF (fig. 2). Depending on the breast volume and the amount of ptosis, the nipple-areola complex (NAC) can be positioned using a superior or inferior pedicle. In breasts with very prominent ptosis and thus a very long pedicle, the NAC may be reconstructed with a free nipple graft. However, this should be regarded as the last reconstructive option for the NAC as free nipple grafts are only perfused by diffusion and are numb.

To shape the contralateral breast, at least 2 techniques are possible: either the contralateral breast is augmented or a breast reduction/mastopexy is performed (fig. 3 a,b,c). Nevertheless, symmetry after implant reconstruction is always difficult to achieve.

\section{Implant-Based Breast Reconstruction (Overweight Patients)}

In overweight patients with small breasts, a periareolar or tennis racquet-like incision can be used for SSM/NSM. The skin flap is usually thicker minimizing complications, and prepectoral placement can be considered as well. However, overweight patients generally have high rates of complications [27]. Using implants, a satisfying result can be achieved, with the breast usually appearing rather flat due the obese body habitus (fig. 4). The reconstructive goal is to provide reasonable symmetry with a satisfactory feel when wearing a bra.

Overweight patients with large breasts usually present with distinct ptosis. Implant reconstruction of the affected breast is usually performed using a Wise-pattern or vertical breast reduction or a reduction pattern of the surgeon's preference. SSM/NSM is performed in a standard fashion, and the implant is usually placed in the retropectoral pocket. Prepectoral placement can be considered in the case of adequate soft tissue cover and is again more frequently per-
Fig. 3. a Breast cancer, right breast, with nipplesparing mastectomy $11 / 2009$. No postoperative radiotherapy; development of capsular fibrosis. b Subpectoral implant exchange und adjusting reduction mammoplasty to the contralateral side 07/2011. c Appealing long-term result 07/2014.

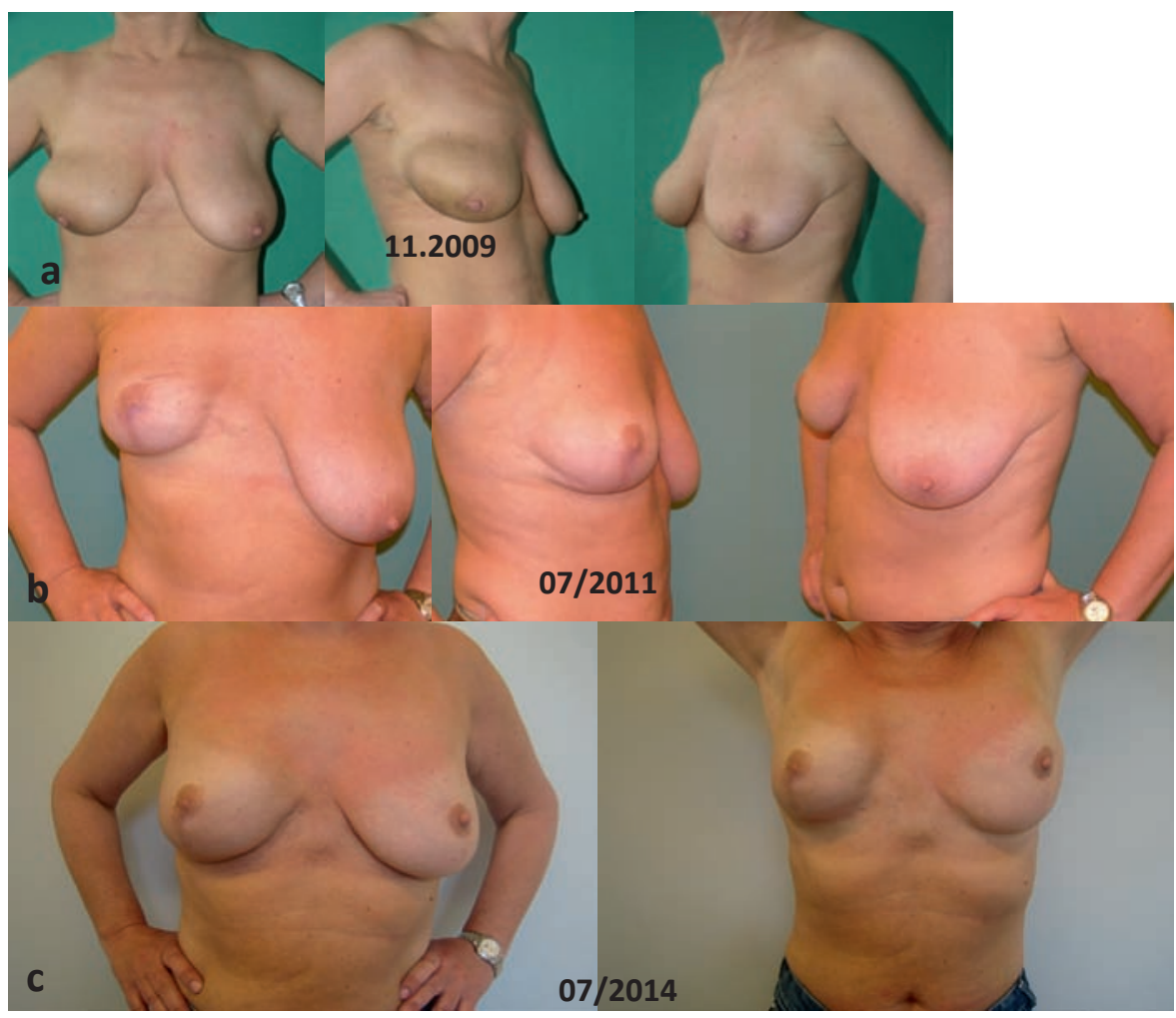




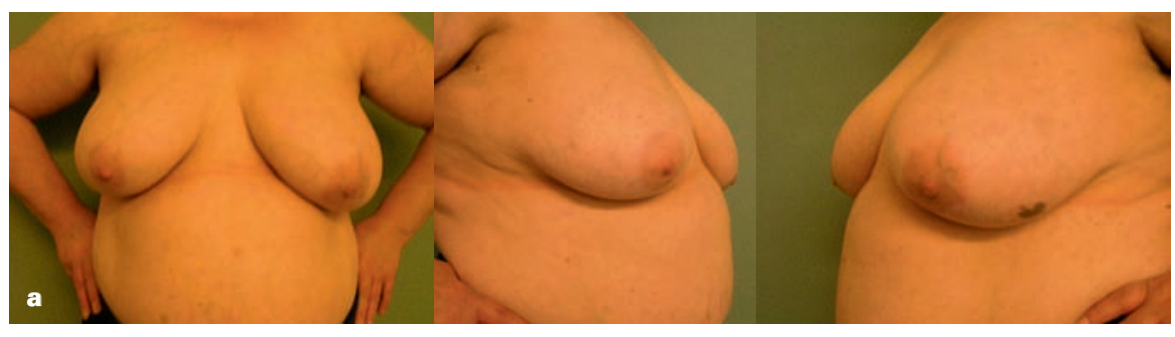

Fig. 4. a 57-year-old patient with large ductal carcinoma in situ, left breast, before surgery. b 6 months after skin-sparing mastectomy using an inverted $\mathrm{T}$ incision, preparation of an inferodermal flap for subpectoral direct-to-implant reconstruction (Allergan Style 410MM, $400 \mathrm{~g}$ ). Simultaneous contralateral adjustment of the right breast. Wound revision first postoperative day, right breast, due to bleeding.
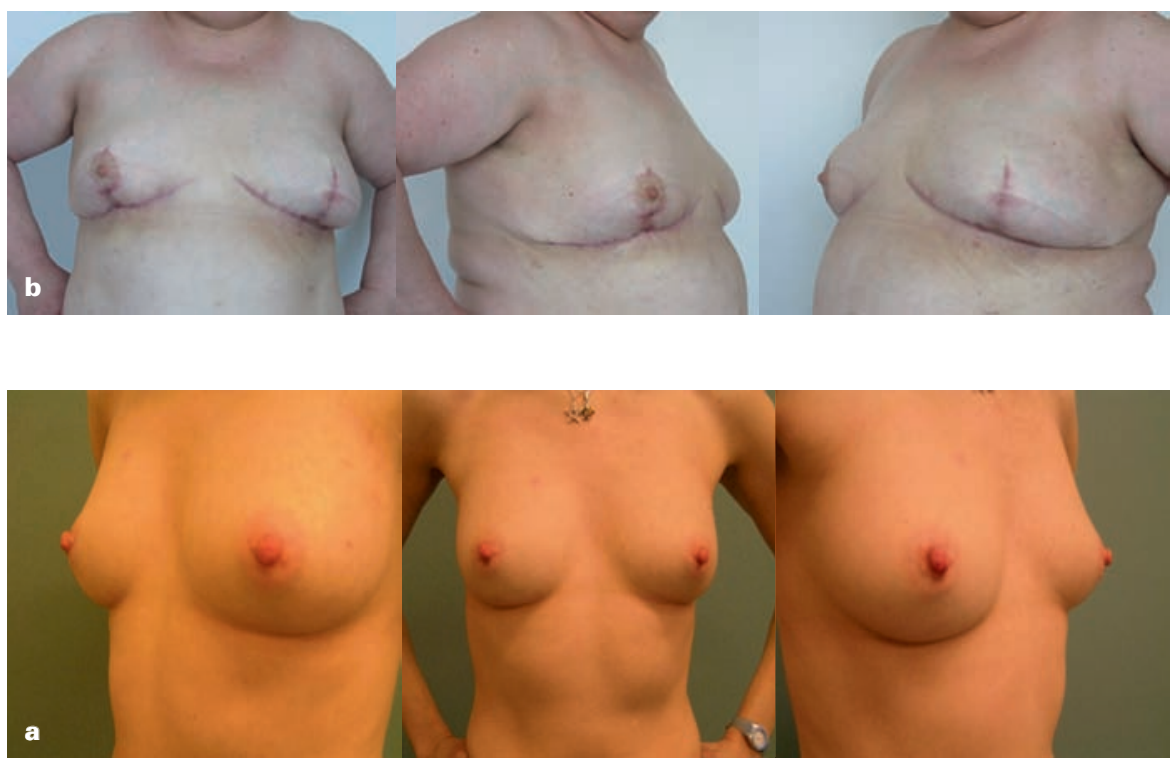

Fig. 5. a 42-year-old patient with multifocal breast cancer, left breast, before surgery.

b 8 months after nipple-sparing mastectomy with immediate latissimus dorsi flap reconstruction, left breast. No implant.

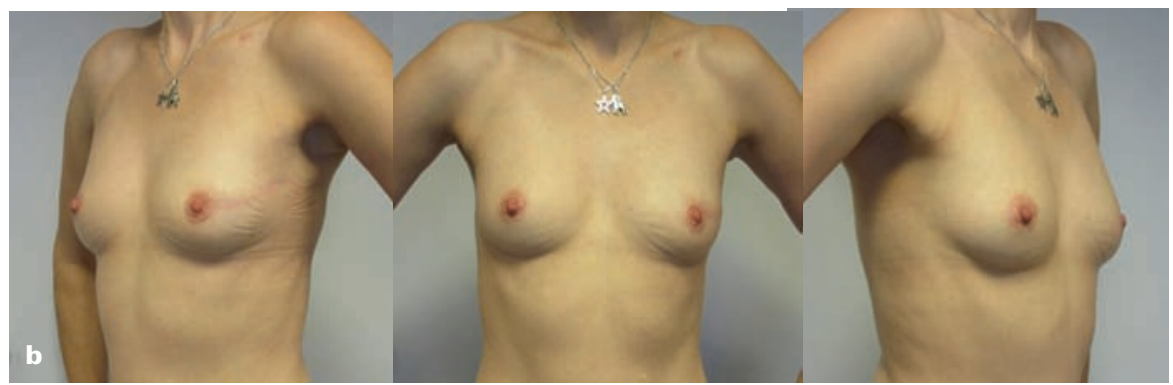

formed. For additional support and implant coverage at the inferior part, a deepithelialized dermal flap can be dissected. Care must be taken regarding tension-free closure in the area where the lower central sutures come together. The contralateral side usually needs a reductive procedure to achieve symmetry. This can either be performed within the same setting or at a later time. Long-term results are satisfying with slight changes in shape over time (fig. 4).

\section{Autologous Breast Reconstruction (Slim Patients)}

As expected, slim patients with small breast present with a rather low body mass index. Consequently, the body habitus may limit the options for autologous breast reconstruction, as some excessive fatty tissue is needed for reconstruction. On the other hand, in most cases, only a small natural breast volume is needed for reconstruction. Thus, a small muscle-sparing transverse rectus abdominis myocutaneous (MS2-TRAM) flap, a deep inferior epigastric perforator (DIEP) flap, or a transverse musculocutaneous gracilis (TMG) flap may provide adequate tissue in these patients to match the opposite breast. In all cases, special care must be taken not to resect too much tissue at the donor site area, hence creating a donor site morbidity due to excessive suture tension. As in all planed microsurgical reconstructive procedures, comorbidities or individual risk factors such as insufficiently treated diabetes or smoking should be reduced as much as possible prior to operation. The latissimus dorsi flap without implant is also a valid option providing good long-term results and can be considered when a certain amount of loose skin is available from the back which has to be deepithelialized (fig. 5).

The challenge in slim patients with larger breasts is that usually not enough donor site tissue is available to reconstruct the affected breast. In these cases, symmetry is often only achieved using an additional implant or a contralateral breast reduction. The armamentarium of autologous procedures is the same as presented previously in slim patients with small breast. The implant is usually placed below the dermal flap or the latissimus dorsi flap. Implant placement below the muscle is favorable in slim patients with thin skin. 
Fig. 6. 53-year-old patient after radical mastectomy and chemotherapy, right breast. $\mathbf{a}$ Before surgery; b 5 days after surgery.

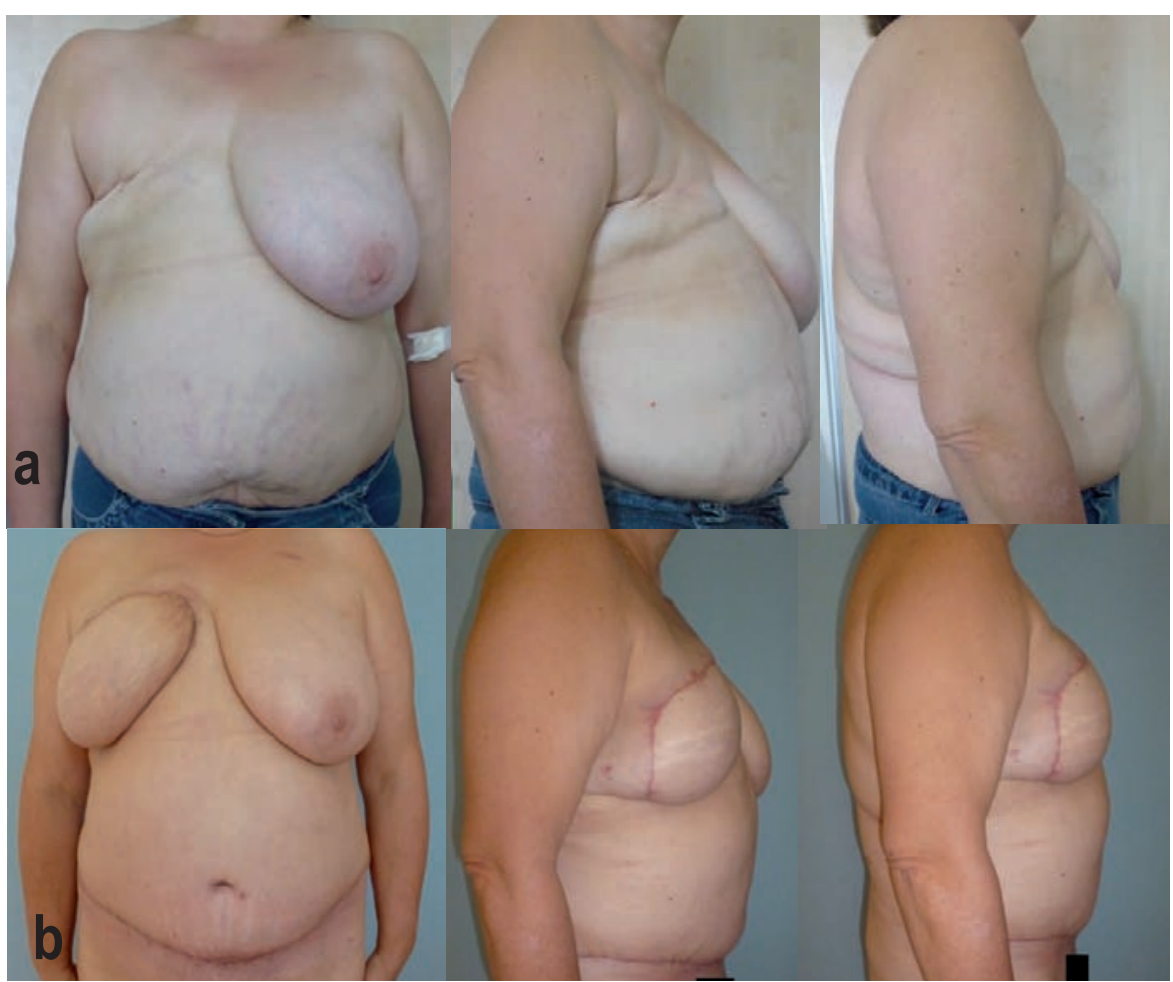

\section{Autologous Breast Reconstruction (Overweight Patients)}

Sufficient donor site tissue is generally not a problem in overweight patients with small breasts. Challenges exit with possible comorbidities resulting in problems with the donor site and the flap. Besides the usual flaps like the pedicled MS2-TRAM, DIEP, TMG, or other free flaps, the latissimus dorsi flap presents a valid and reliable option with usually sufficient tissue for this patient collective; however, it may also be considered as more of a backup option in the case of partial or complete free flap loss.

In overweight patients with large breasts, obesity is no longer a contraindication for autologous breast reconstruction due to refinements in surgical techniques and greater experience with these procedures. The surgeon must be aware of the high risk of fat necrosis and complications due to decreased perfusion [28-30]. One of the major reconstructive challenges in this specific patient collective is the aesthetic shaping of the new breast using the transplanted tissue (fig. 6). The initially transplanted volume is usually much larger than needed even for a large breast. Patients with multiple comorbidities like diabetes, hypertension, very high body mass index, medical history of thrombosis, or heavy smoking should not be considered for autologous or any reconstruction.

\section{Conclusion}

Nowadays, a magnitude of reconstructive choices is available for breast cancer patients. To achieve the most satisfying result for the patient, an exhaustive preoperative consultation discussing all reconstructive options is crucial. The expectations of the patient must be considered and discussed bearing in mind reconstructive restrictions regarding body habitus and comorbidities. Using microsurgical techniques, profound experience in the field of reconstructive microsurgery (ideally with a 24 -h available microsurgical emergency team) is an important part of successful interdisciplinary treatment in breast reconstruction. Difficulties and challenges need to be addressed to find the best approach for each individual patient. A broad reconstructive as well as oncologic education, like the AWO-gyn breast surgeon training concept in Germany, is necessary to link all these factors and support our patients [31].

\section{Disclosure Statement}

None of the authors have any conflict of interest to disclose.

All costs were handled by the hospital and the authors themselves. No funding from third parties was received.

\section{References}

1 RKI, Robert-Koch-Institut: Evaluation of Cancer Incidence in Germany (2007-2010). www.rki.de/DE/Home/ homepage_node.html; last accessed on 5 August 2017.

2 Romics L, Jr, Chew BK, Weiler-Mithoff E, et al.: Tenyear follow-up of skin-sparing mastectomy followed by immediate breast reconstruction. Br J Surg 2012;99: 799-806.
3 Mallon P, Feron JG, Couturaud B, et al.: The role of nipple-sparing mastectomy in breast cancer: a comprehensive review of the literature. Plast Reconstr Surg 2013;131:969-984.

4 Gerber B, Krause A, Dieterich M, et al.: The oncological safety of skin sparing mastectomy with conserva- tion of the nipple-areola complex and autologous reconstruction: an extended follow-up study. Ann Surg 2009;249:461-468.

5 Kronowitz SJ, Robb GL: Radiation therapy and breast reconstruction: a critical review of the literature. Plast Reconstr Surg 2009;124:395-408. 
6 Overgaard M, Nielsen HM, Overgaard J: Is the benefit of postmastectomy irradiation limited to patients with four or more positive nodes, as recommended in international consensus reports? A subgroup analysis of the DBCG 82 b\&c randomized trials. Radiother Oncol 2007;82:247-253.

7 EBCTCG: Overview of the Randomized Trials of Radiotherapy in Early Breast Cancer. Educational Book ASCO, 2007, p. 3-6.

8 AGO Breast Commission: Diagnosis and Treatment of Patients with Primary and Metastatic Breast Cancer. Recommendations 2017. www.ago-online.de; accessed June 2017.

9 Weiser MR, Montgomery LL, Susnik B, et al.: Is routine intraoperative frozen-section examination of sentinel lymph nodes in breast cancer worthwhile? Ann Surg Oncol 2000;7:651-655.

10 Krishnamurthy S, Meric-Bernstam F, Lucci A, et al.: A prospective study comparing touch imprint cytology, frozen section analysis, and rapid cytokeratin immunostain for intraoperative evaluation of axillary sentinel lymph nodes in breast cancer. Cancer 2009;115:1555-1562.

11 Vanderveen KA, Ramsamooj R, Bold RJ: A prospective, blinded trial of touch prep analysis versus frozen section for intraoperative evaluation of sentinel lymph nodes in breast cancer. Ann Surg Oncol 2008;15:2006-2011.

12 Lentz R, Ng R, Higgins SA, et al.: Radiation therapy and expander-implant breast reconstruction: an analysis of timing and comparison of complications. Ann Plast Surg 2013;71:269-273.

13 Nava MB, Pennati AE, Lozza L, et al.: Outcome of different timings of radiotherapy in implant-based breast reconstructions. Plast Reconstr Surg 2011;128:353-359.

14 Cordeiro PG, Albornoz CR, McCormick B, et al.: What is the optimum timing of post-mastectomy radiotherapy in two-stage prosthetic reconstruction: radiation to the tissue expander or permanent implant? Plast Reconstr Surg 2015;135:1509-1517.

15 Peled AW, Foster RD, Esserman LJ, et al.: Increasing the time to expander-implant exchange after postmastectomy radiation therapy reduces expander-implant failure. Plast Reconstr Surg 2012;130:503-509.

16 Nahabedian MY, Tsangaris T, Momen B, Manson PN: Infectious complications following breast reconstruction with expanders and implants. Plast Reconstr Surg 2003;112:467-476.

17 Foster RD, Hansen SL, Esserman LJ, et al.: Safety of immediate transverse rectus abdominis myocutaneous breast reconstruction for patients with locally advanced disease. Arch Surg 2005;140:196-198; discussion 199-200.

18 Rogers NE, Allen RJ: Radiation effects on breast reconstruction with the deep inferior epigastric perforator flap. Plast Reconstr Surg 2002;109:1919-1924; discussion 1925-1926.

19 Disa JJ, Ad-El DD, Cohen SM, et al.: The premature removal of tissue expanders in breast reconstruction. Plast Reconstr Surg 1999;104:1662-1665.

20 Gerber B, Marx M, Untch M, Faridi A: Breast reconstruction following cancer treatment. Dtsch Arztebl Int 2015;112:593-600.

21 Dieterich M, Faridi A: Biological matrices and synthetic meshes used in implant-based breast reconstruction - a review of products available in Germany. Geburtshilfe Frauenheilkd 2013;73:1100-1106.

22 American Society of Plastic Surgeons: Plastic Surgery Statistics Report; www.plasticsurgery.org/documents/ News/Statistics/2016/plastic-surgery-statistics-full-report2016.pdf; last accessed 7 August 2017.

23 Cabalag MS, Rostek M, Miller GS, et al.: Alloplastic adjuncts in breast reconstruction. Gland Surg 2016;5: 158-173.
24 Dieterich M, Angres J, Stubert J, et al.: Patient-reported outcomes in implant-based breast reconstruction alone or in combination with a titanium-coated polypropylene mesh - a detailed analysis of the BREAST-Q and overview of the literature. Geburtshilfe Frauenheilkd 2015;75:692-701.

25 Martin L, O'Donoghue JM, Horgan K, et al.: Acellular dermal matrix (ADM) assisted breast reconstruction procedures: joint guidelines from the Association of Breast Surgery and the British Association of Plastic, Reconstructive and Aesthetic Surgeons. Eur J Surg Oncol 2013;39:425-429.

26 Gurunluoglu R, Gurunluoglu A, Williams SA, Tebockhorst S: Current trends in breast reconstruction: survey of American Society of Plastic Surgeons 2010. Ann Plast Surg 2013;70:103-110.

27 Davies K, Allan L, Roblin P, et al.: Factors affecting post-operative complications following skin sparing mastectomy with immediate breast reconstruction. Breast 2011;20:21-25

28 Garvey PB, Buchel EW, Pockaj BA, et al.: The deep inferior epigastric perforator flap for breast reconstruction in overweight and obese patients. Plast Reconstr Surg 2005;115:447-457.

29 Chang DW, Wang B, Robb GL, et al.: Effect of obesity on flap and donor-site complications in free transverse rectus abdominis myocutaneous flap breast reconstruction. Plast Reconstr Surg 2000;105:1640-1648.

30 Nahabedian MY, Momen B, Galdino G, Manson PN: Breast reconstruction with the free TRAM or DIEP flap: patient selection, choice of flap, and outcome. Plast Reconstr Surg 2002;110:466-475; discussion 476-477.

31 Dieterich M, Dieterich H, Nestle-Kramling C, et al. Certification of breast surgeons in Germany - AWOgyn curriculum in an International comparison. Geburtsh Frauenheilk 2009;69:987-991. 